\title{
BMJ Open Educating hospital patients to prevent falls: protocol for a scoping review
}

\author{
Hazel Heng, ${ }^{1}{ }^{1}$ Dana Jazayeri, ${ }^{1}$ Louise Shaw, ${ }^{1}$ Debra Kiegaldie, ${ }^{2,3}$ Anne-Marie Hill, ${ }^{4}$ \\ Meg E Morris ${ }^{1,5}$
}

To cite: Heng H, Jazayeri D, Shaw L, et al. Educating hospital patients to prevent falls: protocol for a scoping review. BMJ Open 2019;9:e030952. doi:10.1136/ bmjopen-2019-030952

- Prepublication history and additional material for this paper are available online. To view these files, please visit the journal online (http://dx.doi. org/10.1136/bmjopen-2019030952).

Received 09 April 2019 Revised 12 August 2019 Accepted 02 September 2019

Check for updates

(c) Author(s) (or their employer(s)) 2019. Re-use permitted under CC BY-NC. No commercial re-use. See rights and permissions. Published by BMJ.

${ }^{1}$ La Trobe Centre for Sport and Exercise Medicine Research, La Trobe University, Bundoora, Victoria, Australia

${ }^{2}$ Healthscope and Faculty of Health Science, Youth and Community Studies, Holmesglen Institute of TAFE Morrabbin

Campus, Moorabbin, Victoria, Australia

${ }^{3}$ Faculty of Medicine Nursing and Health Sciences, Monash University, Clayton, Victoria,

Australia

${ }^{4}$ School of Physiotherapy and Exercise Science, Curtin University, Perth, Western Australia, Australia ${ }^{5}$ North Eastern Rehabilitation Centre, Healthscope Ltd, Ivanhoe, Victoria, Australia

Correspondence to

Hazel Heng;

h.heng@latrobe.edu.au

\section{ABSTRACT}

Introduction Falls prevention in hospitals is an ongoing challenge worldwide. Despite a wide variety of recommended falls mitigation strategies, few have strong evidence for effectiveness in reducing falls and accompanying injuries. Patient education programmes that promote engagement and enable people to understand their heightened falls risk while hospitalised are one approach. The aim of this scoping review is to examine the content, design and outcomes of patient education approaches to hospital falls prevention. As well as critiquing the role of patient education in hospital falls prevention, strategies that can be used in clinical practice shall be recommended.

Methods and analysis The analysis will apply the methodological framework developed by Arksey and O'Malley and refined by the Joanna Briggs Institute. An initial limited search of Cumulative Index to Nursing and Allied Health Literature (CINAHL) and PubMed will be completed to identify keywords and index terms. A developed search strategy of Medical Subject Headings and text words will be conducted of PubMed, CINAHL, Cochrane Central Register of Controlled Trials, Allied and Complementary Medicine Database, PsychINF0, Education Resources Information Center and grey literature databases from January 2008 to current. The reference lists of included articles will be hand searched for additional studies. Two reviewers will screen the titles and abstracts independently and analyse the full text of potential articles based on the inclusion and exclusion criteria. The data will be extracted using a structured data form. Thematic analysis and numerical synthesis of the data will be conducted, and key themes will be identified. Ethics and dissemination Results of this scoping review will illuminate the designs and outcomes of patient education research for hospital falls prevention in the current literature. It is anticipated that the findings will highlight best-practice educational design to inform the development of future patient-focused education for falls prevention. Study findings will be presented at relevant conferences and public forums, and published in peer-reviewed journals. Ethics approval is not required.

\section{BACKGROUND}

Falls are an ongoing serious issue within hospitals worldwide. They are linked with increased length of stay, poor functional outcomes and an increased risk of institutionalisation. ${ }^{1-3}$ There is an associated rise
Strengths and limitations of this study

The critical scoping review maps key elements of effective patient education to prevent falls and sheds light on which educational interventions help patients themselves mitigate falling while in hospital.

- The review will conduct a quality assessment of patient education programmes in the literature.

- The review will be limited to studies in English conducted from January 2008.

in costs for hospitals after the occurrence of an in-patient fall, regardless of whether injuries are sustained. ${ }^{45}$ Due to the significant cost to health and well-being, a growing body of research has investigated the causes of falls in hospitals. Some key risk factors include a history of past falls, gait instability, balance impairment, cognitive impairment, multimorbidity, polypharmacy and urinary frequency. ${ }^{6}$ Falls prevention in hospitals nevertheless remains a difficult task. Whereas single interventions have not been very successful in reducing hospital fall rates, ${ }^{28-11}$ multifactorial interventions might be effective for some individuals, particularly in subacute and aged care settings. ${ }^{212-14}$ Nevertheless, despite careful implementation of different strategies such as education, environmental modifications, mobility aids, alarms and physical therapies ${ }^{21516}$ as defined in the ProFaNE classification, ${ }^{17}$ it is unclear how falls can be routinely prevented in hospitals or the relative contribution of patient education.

Emerging research indicates that patient-related factors can influence the frequency and severity of hospital falls. ${ }^{18}$ In particular, falls knowledge and insight of patients into their own falls risk is a key determinant of hospital falls. $^{19}$ A qualitative study investigated patients' views of their falls risk and observed a disparity between their perceptions and the actual risk when in hospital. ${ }^{20}$ Patients sometimes took unnecessary risks, such as getting out of bed and toileting without assistance, and they did not always engage fully with falls 
prevention strategies. ${ }^{21}$ This was particularly the case for hospitalised patients with dementia, delirium and other cognitive impairments. ${ }^{22}$

While many falls prevention strategies involve some form of education aimed at increasing patient knowledge, ${ }^{2}$ few have designed the intervention based on behaviour change models and educational principles, or have provided adequate descriptions of these. ${ }^{23}{ }^{24}$ One randomised controlled trial delivered an individualised education programme which resulted in a significant reduction in the rate of hospital falls and fall-related injuries. ${ }^{12}$ The study investigated the provision of a patient education programme informed by adult learning and health behaviour change principles, with individually tailored follow-up sessions provided by an educator. ${ }^{12}$ Well-designed patient education also has the potential to increase adherence to falls prevention strategies, thereby reducing slips, trips and falls. ${ }^{25} 26$

Given that patient education has the potential to reduce hospital falls and injuries, more attention needs to be directed towards the structure of patient education programmes, how they are delivered and how to measure their effectiveness. Depending on the characteristics of the target population, further considerations need to be made. For example, people with impaired cognition or of different languages and cultural backgrounds might require different approaches. ${ }^{22}{ }^{27}$ Likewise, those with a known history of frequent falling may respond to particular methods of falls prevention.

Our scoping review shall provide a new and detailed analysis of the benefits and limitations of patient education strategies for mitigation of falls in acute hospitals and subacute settings such as rehabilitation units. Even though a Cochrane review of falls prevention interventions was conducted by Cameron and colleagues, ${ }^{2}$ that analysis was restricted to adults over 65 years of age or studies with a mean age greater than 65 years. The Cameron review excluded interventions that took place in hospital emergency departments or hospital outpatient settings. ${ }^{2}$ It did not provide details on the exact methods used to educate hospitalised patients on how to prevent falling or details on the mode of delivery, such as handouts, posters, multimedia or face-to-face discussions. The current scoping review shall address these gaps, as well as including more recent data published since the Cameron review.

We shall conduct a scoping review of the literature to map evidence, given the paucity of published reports on patient education to reduce hospital falls and the wide variations in the interventions and methodologies used in existing studies. According to the Joanna Briggs Institute (JBI), scoping reviews are particularly helpful for assembling evidence from disparate or heterogeneous sources. ${ }^{28}{ }^{29}$ Scoping reviews also "... provide a map of what evidence has been produced as opposed to seeking only the best available evidence to answer a particular question related to policy and practice". ${ }^{28}$ They are very helpful for identifying gaps in the literature and for mapping key concepts underpinning a research area and elucidating working definitions. ${ }^{28}{ }^{30}$ Our scoping review will therefore bring together existing and emerging evidence from a broad range of sources with different levels of quality, to crystallise the key concepts underpinning this research area and to clarify working definitions.

This scoping review aims to examine the literature regarding the use and effectiveness of patient education in hospitals to reduce the risk of falls and injuries arising from falls. The specific objectives are to: (1) examine patient education interventions for falls prevention in hospitals; (2) evaluate the design of patient hospital falls prevention education programmes; and (3) identify the outcome measures used and, where applicable, critique their clinimetric properties.

\section{METHODS AND ANALYSIS}

The methodological framework developed by Arksey and $\mathrm{O}^{\prime} \mathrm{Malley}^{30}$ and refined by the Joanna Briggs Institute ${ }^{29}$ will be used along with the Preferred Reporting Items for Systematic Reviews and Meta-Analyses Extension for Scoping Reviews (PRISMA-ScR). ${ }^{31}$ This framework has five stages: (1) identifying the research question; (2) identifying relevant studies; (3) study selection; (4) data charting and (5) collating, summarising and reporting the results. ${ }^{29} 30$ Each stage will be discussed in detail below.

\section{Patient and public involvement}

This protocol was designed with patient involvement at each step, and consumers will be involved in the review and its dissemination. Consumer representatives were invited to comment on the design and contributed to the editing of this document.

\section{Stage 1: identifying the research question}

A scoping review aims to identify where the strongest evidence exists as well as opportunities for further research. To identify gaps in knowledge by summarising the breadth of evidence, the research question should be broad. The overarching question developed for this review is "What patient education research has been implemented for falls prevention within hospitals?" Further secondary questions have also been identified to guide the review: (1) What content does the patient education include? (2) Is the education design based on best-practice educational principles and/or behaviour change models? (3) What are the outcomes of patient education research?

\section{Stage 2: identifying relevant studies}

\section{Eligibility criteria}

JBI recommends defining the following elements: 'population', 'concept' and 'context' which will guide the inclusion and exclusion criteria. ${ }^{29}$ For this review, the population is defined as adult patients (18 years or older) who are hospitalised. Studies that involve education delivered to families of cognitively impaired individuals will also be included. The key concept in this scoping review is patient education 
for falls prevention. This includes any studies that assess falls prevention interventions with an aspect of patient education, such as multifactorial interventions. Studies will only be included if they are in a hospital setting (eg, acute, subacute, rehabilitation) which is the context of the review. Studies will be excluded if they are non-empirical, set in residential care or the community or involve paediatric populations. Investigations solely on clinician education will also be excluded. To capture the appropriate range of literature, all research study designs are eligible, including quantitative, qualitative and mixed-methods studies.

\section{Search strategy}

A three-step approach will be used to search for published and unpublished studies. First, an initial limited search of Cumulative Index to Nursing and Allied Health Literature (CINAHL) and PubMed will be conducted. Articles identified will be analysed for words contained in the title and abstract and of the index terms used to describe the articles. Medical subject headings and words related to patient education and falls prevention in hospitals will be developed by a qualified librarian in conjunction with previously identified key words and index terms. A second search will then be undertaken across the following databases: PubMed, CINAHL, the Cochrane Central Register of Controlled Trials, Allied and Complementary Medicine Database, PsychINFO and Education Resources Information Center. This process will be iterative to ensure all relevant search terms are captured. The search for unpublished studies will include Trove and ProQuest Theses and Dissertations Global. Articles will be limited to the English language and the last 10 years from January 2008 to current. This is to ensure that the data are up to date as hospital systems have changed over time and new falls prevention programmes are being implemented. The search strategy for CINAHL can be found in the online supplementary file 1. Finally, the reference list of all identified reports and articles will be searched for additional studies.

\section{Stage 3: study selection}

Two reviewers will independently screen the title and abstracts of retrieved papers to identify potentially relevant articles. The full text of the identified papers will be obtained and assessed by two independent reviewers. Any discrepancies will be resolved through discussions, and if required, consensus will be achieved by seeking input from a third reviewer. Covidence, a web-based platform which streamlines the production of systematic reviews, will be used to assist the screening of articles. Results of the search strategy, including the final included and excluded studies will be presented in a PRISMA flow diagram. ${ }^{31}$

\section{Stage 4: data charting}

A data extraction chart will be used. Abstracted data will include the following items: author, year of publication, country of origin, aims of the study, patient characteristics, hospital setting, education design characteristics, research methodology, measurement tools and reported outcomes. Additional variables may be identified following complete review of the full text. The same two reviewers will independently chart the data. Once data have been extracted, the quality of patient education will be charted using a quality metric. The metric is a tool created by Kiegaldie and Farlie ${ }^{32}$ to assess the quality of education programmes delivered to health professionals in the context of falls prevention research. This review will use a version modified by the authors which excludes items specific to clinician education and can be found in the online supplementary file 1 . For the purposes of this review, the 'learner' is the patient and the 'co-learners' are the families or carers of cognitively impaired patients. As formal quality assessment of articles is generally not required due to the nature of scoping reviews, ${ }^{29} 30$ a broad overview of the research quality of the studies will be included instead.

\section{Stage 5: collating, summarising and reporting the results}

The data extracted will be summarised qualitatively via thematic analysis, and quantitative data will be summarised using numerical counts. For this scoping review, we decided to use expert reviewers rather than commercially available software to analyse the results and identify key themes. An iterative process of identifying new categories and themes will arise through ongoing analysis. Where appropriate, interventions will be categorised as: (1) approaches that directly educate patients; (2) environmental modifications where patient education is involved; (3) systems, policies and procedures that include patient education and (4) consumer materials for falls prevention. These interventions will be classified according to the mode of delivery, such as through face-toface discussions, videos, brochures, posters, handouts or multimedia. Single interventions or multimodal methods of patient falls education will be investigated. Links between the quality of patient education and outcome measures (falls or educational) will also be identified and reported. If reported, educational design principles will be evaluated for best practice based on the recommendations of ProFaNE. ${ }^{33}$ Some of these recommendations include raising awareness of falls, promoting positive self-identity and encouraging self-management. ${ }^{33} 34$

We shall distinguish between effective and non-effective fall prevention educational programmes by examining a combination of outcomes, such as the number of fallers divided by the total number of patients for a given unit (risk of falls); the rate of falls over a given time taking into account exposure, such as the number of falls per occupied bed days, expressed as falls per 1000 bed days (falls rate). Moreover, we shall examine effectiveness of falls prevention in relation to the quality of the educational interventions as reflected by patient knowledge, compliance and satisfaction. Results will be presented as tables, charts and diagrams where appropriate, to allow for easy comparison. Following synthesis and analysis of the data, this scoping review will be able to identify the strengths and limitations of existing methods of patient education and areas for future research. 


\section{ETHICS AND DISSEMINATION}

This scoping review does not require ethics approval as data will be obtained through review of existing literature. Study findings will be presented at relevant conferences and public forums, and published in peer-reviewed journals.

The link between effective patient education, empowerment and adherence, as well as effective process implementation should not be underestimated. By drawing on this link, we hope to inform the direction of future research to empower patients to recognise their falls risk and promote engagement with falls prevention strategies while they are in hospital.

Contributors All authors contributed to the preparation, drafting and editing of this scoping review protocol. HH, LS, MEM and DK designed the protocol. HH wrote the first draft, and DK, MEM, DJ and A-MH critically appraised and revised the manuscript. All authors read and approved the final version of the manuscript.

Funding This scoping review is being conducted as a part of an NHMRC funded grant partnership (\#GNT1152853) which aims to use implementation science principles to enable both clinicians and patients to better mitigate future risk of hospital falls and to reduce falls rates. The partnership is between NHMRC the Healthscope private hospital network, Holmesglen Institute and Australian universities.

Competing interests All authors report a grant from the National Health and Medical Research Council Australia to fund this project, during the conduct of the study.

Patient consent for publication Not required.

Provenance and peer review Not commissioned; externally peer reviewed.

Open access This is an open access article distributed in accordance with the Creative Commons Attribution Non Commercial (CC BY-NC 4.0) license, which permits others to distribute, remix, adapt, build upon this work non-commercially, and license their derivative works on different terms, provided the original work is properly cited, appropriate credit is given, any changes made indicated, and the use is non-commercial. See: http://creativecommons.org/licenses/by-nc/4.0/.

\section{REFERENCES}

1. Oliver D, Healey F, Haines TP. Preventing falls and fall-related injuries in hospitals. Clin Geriatr Med 2010;26:645-92.

2. Cameron ID, Dyer SM, Panagoda CE, et al. Interventions for preventing falls in older people in care facilities and hospitals. Cochrane Database Syst Rev 2018;27.

3. Lerdal A, Sigurdsen LW, Hammerstad H, et al. Associations between patient symptoms and falls in an acute care hospital: a crosssectional study. J Clin Nurs 2018;27:1826-35.

4. Morello RT, Barker AL, Watts JJ, et al. The extra resource burden of in-hospital falls: a cost of falls study. Med J Aust 2015;203:367.

5. Heinrich S, Rapp K, Rissmann U, et al. Cost of falls in old age: a systematic review. Osteoporos Int 2010;21:891-902.

6. Oliver D, Daly F, Martin FC, et al. Risk factors and risk assessment tools for falls in hospital in-patients: a systematic review. Age Ageing 2004;33:122-30.

7. Deandrea S, Bravi F, Turati F, et al. Risk factors for falls in older people in nursing homes and hospitals. A systematic review and meta-analysis. Arch Gerontol Geriatr 2013;56:407-15.

8. Haines TP, Hill A-M, Hill KD, et al. Patient education to prevent falls among older hospital inpatients. Arch Intern Med 2011;171:516-24.

9. Michalek C, Wehling M, Schlitzer J, et al. Effects of "Fit fOR The Aged" (FORTA) on pharmacotherapy and clinical endpoints - a pilot randomized controlled study. Eur J Clin Pharmacol 2014;70:1261-7.
10. Treacy D, Schurr K, Lloyd B, et al. Additional standing balance circuit classes during inpatient rehabilitation improved balance outcomes: an assessor-blinded randomised controlled trial. Age Ageing 2015;44:580-6.

11. Haines TP, Bell RAR, Varghese PN. Pragmatic, cluster randomized trial of a policy to introduce low-low beds to hospital wards for the prevention of falls and fall injuries. J Am Geriatr Soc 2010;58:435-41.

12. Hill $\mathrm{AM}$, McPhail SM, Waldron $\mathrm{N}$, et al. Fall rates in hospital rehabilitation units after individualised patient and staff education programmes: a pragmatic, stepped-wedge, cluster-randomised controlled trial. Lancet 2015;385:2592-9.

13. Barker AL, Morello RT, Wolfe R, et al. 6-PACK programme to decrease fall injuries in acute hospitals: cluster randomised controlled trial. BMJ 2016;352.

14. Beck AM, Christensen AG, Hansen BS, et al. Multidisciplinary nutritional support for undernutrition in nursing home and home-care: a cluster randomized controlled trial. Nutrition 2016;32:199-205.

15. Coussement J, De Paepe L, Schwendimann R, et al. Interventions for preventing falls in acute- and chronic-care hospitals: a systematic review and meta-analysis. J Am Geriatr Soc 2008;56:29-36.

16. Cumming RG, Sherrington C, Lord SR, et al. Cluster randomised tria of a targeted multifactorial intervention to prevent falls among older people in hospital. BMJ 2008;336:758-60.

17. Lamb SE, Becker C, Gillespie LD, et al. Reporting of complex interventions in clinical trials: development of a taxonomy to classify and describe fall-prevention interventions. Trials 2011;12:125.

18. Shuman C, Liu J, Montie M, et al. Patient perceptions and experiences with falls during hospitalization and after discharge. Appl Nurs Res 2016;31:79-85.

19. Haines TP, Lee D-CA, O'Connell B, et al. Why do hospitalized older adults take risks that may lead to falls? Health Expect 2015;18:233-49.

20. Carroll DL, Dykes PC, Hurley AC. Patients' perspectives of falling while in an acute care hospital and suggestions for prevention. Appl Nurs Res 2010;23:238-41.

21. Dykes PC, Carroll DL, Hurley AC, et al. Why do patients in acute care hospitals fall? can falls be prevented? J Nurs Adm 2009;39:299-304.

22. Oliver D, Connelly JB, Victor CR, et al. Strategies to prevent falls and fractures in hospitals and care homes and effect of cognitive impairment: systematic review and meta-analyses. BMJ 2007;334.

23. Michie S, Fixsen D, Grimshaw JM, et al. Specifying and reporting complex behaviour change interventions: the need for a scientific method. Implement Sci 2009;4.

24. McHugh S, Sinnott C, Racine E, et al. 'Around the edges': using behaviour change techniques to characterise a multilevel implementation strategy for a fall prevention programme. Implement Sci 2018;13.

25. Falvo D. Effective patient education: a guide to increased adherence. 4th edn. Sudbury, MA: Jones \& Bartlett Publishers, 2010.

26. D-CA L, Pritchard E, McDermott F, et al. Falls prevention education for older adults during and after hospitalization: a systematic review and meta-analysis. Health Educ J 2014;73:530-44.

27. Jang $\mathrm{H}$, Clemson $\mathrm{L}$, Lovarini $\mathrm{M}$, et al. Cultural influences on exercise participation and fall prevention: a systematic review and narrative synthesis. Disabil Rehabil 2016;38:724-32.

28. Peters MDJ, Godfrey C, Mclnerney P, et al. Chapter 11: Scoping Reviews. In: Aromataris E, Munn Z, eds. Joanna Briggs Institute reviewer's manual. The Joanna Briggs Institute, 2017.

29. Peters MDJ, Godfrey CM, Khalil H, et al. Guidance for conducting systematic scoping reviews. Int J Evid Based Healthc 2015;13:141-6.

30. Arksey H, O'Malley L. Scoping studies: towards a methodological framework. Int J Soc Res Methodol 2005;8:19-32.

31. Tricco AC, Lillie E, Zarin W, et al. PRISMA extension for scoping reviews (PRISMA-ScR): checklist and explanation. Ann Intern Med 2018;169:467-73.

32. Kiegaldie DJ, Farlie MK. Using education interventions in falls research: a framework for evidence-based education design. 2nd edn. Australia: Holmesglen Institute, 2019.

33. Yardley L, Beyer N, Hauer K, et al. Recommendations for promoting the engagement of older people in activities to prevent falls. Qual Saf Health Care 2007;16:230-4.

34. Nyman SR. Psychosocial issues in engaging older people with physical activity interventions for the prevention of falls. Can J Aging 2011;30:45-55. 